\title{
From distribution to replication in cooperative systems with active membranes: A frontier of the efficiency
}

\author{
Luis Valencia-Cabrera, David Orellana-Martín, Miguel Á. Martínez-del-Amor, Agustín Riscos- \\ Núñez, Mario J. Pérez-Jiménez
}

Research Group of Natural Computing, Department of Computer Science and Artificial Intelligence, Universidad de Sevilla, Sevilla, 41012, Spain

Keywords:

Membrane Computing

Active membranes

Minimal cooperation

Mitosis

Computational complexity

The $\mathbf{P}$ versus NP problem

\section{A B S T R A C T}

P systems with active membranes use evolution, communication, dissolution and division (or separation) rules. They do not use cooperation neither priorities, but they have electrical charges associated with membranes, which can be modified by rule applications. The inspiration comes from the behaviour of living cells, who "compute" with their proteins in order to obtain energy, create components, send information to other cells, kill themselves (in a process called apoptosis), and so on. In these models, mitosis is simulated by division rules (for elementary and non-elementary membranes) and meiosis, that is, membrane fission inspiration, is captured in separation rules. The parent's objects are replicated into both child membranes when a division occurs, while in the case of separation, objects are distributed (according to a prefixed partition). In both cases, active membranes have been proved to be too powerful for solving computationally hard problems in an efficient way. Due to this, polarizationless $\mathrm{P}$ systems with active membranes have been widely studied from a complexity point of view.

Evolution rules simulate the transformation of components in membranes, but it is well known that in Biology elements interact with each other in order to obtain new components. In this paper, (restricted) cooperation in object evolution rules is considered, and the efficiency of the corresponding models is studied.

\section{Introduction}

Membrane Computing is a distributed parallel computing paradigm inspired by the way the living cells process chemical substances, energy and information. The processor units in the basic model are abstractions of biological membranes, selectively permeable barriers which give cells their outer boundaries (plasma membranes) and their inner compartments (organelles). They control the flow of information between cells and the movement of substances into and out of cells, and they are also involved in the capture and release of energy. Biological membranes play an active part in the life of the cell. In fact, the passing of a chemical substance through a biological membrane is often implemented by an interaction between the membrane itself and the protein channels present in it. During this interaction, both the chemical substance and the membrane can be modified, at least locally. 
Mitosis is a process by which two or more cells are produced/generated from one cell that could be considered as the "mother". Several cell division inspired mechanisms were introduced in Membrane Computing. Specifically, P systems with active membranes [7] are non-cooperative systems that incorporate the mitosis based mechanisms by means of membrane division rules. By applying this kind of rules, under the influence of the object triggering it, the membrane is divided into two membranes and that object is replaced in the two new ones by possibly new objects, while the remaining objects are duplicated in both newly created membranes. These models are universal (they are equivalent in power to deterministic Turing machines) and they have the ability to provide efficient solutions to computationally hard problems, by making use of an exponential workspace created in a polynomial time (often, in linear time). Moreover, PSPACE-complete problems can be efficiently solved by families of $P$ systems with active membranes which use division for elementary and non-elementary membranes. This paper deals with $\mathrm{P}$ systems with active membranes where electrical charges are removed, separation rules are used as a mechanism to generate an exponential number of membranes in polynomial time, and a restricted minimal cooperation in object evolution rules is considered. The efficiency of these new models is studied.

The paper is organized as follows. Next section briefly describes some preliminaries in order to make the work selfcontained. In Section 3, syntax and semantics of polarizationless P systems with active membranes by using membrane division rules or membrane separation rules are introduced. Definition of Recognizer membrane systems is recalled in Section 4, as a framework to provide efficient solutions to decision problems. The concept of bounded minimal cooperation in object evolution rules is explained in Section 5. Next section is dedicated to show the limits of the computational efficiency of the polarizationless $\mathrm{P}$ systems with active membranes, separation rules and bounded minimal cooperation in object evolution rules. The computational complexity frontier between these systems and of polarizationless P systems with active membranes, division rules only for elementary membranes, minimal cooperation and without dissolution rules is established in Section 7.

The paper ends with some open problems and concluding remarks.

\section{Preliminaries}

An alphabet $\Gamma$ is a non-empty set and their elements are called symbols. A string $u$ over $\Gamma$ is an ordered finite sequence of symbols, that is, a mapping from a natural number $n \in \mathbb{N}$ onto $\Gamma$. The number $n$ is called the length of the string $u$ and it is denoted by $|u|$, that is, the length of a string is the number of occurrences of symbols that it contains. The empty string (with length 0 ) is denoted by $\lambda$. The set of all strings over an alphabet $\Gamma$ is denoted by $\Gamma^{*}$. A language over $\Gamma$ is a subset of $\Gamma^{*}$.

A multiset over an alphabet $\Gamma$ is an ordered pair $(\Gamma, f)$ where $f$ is a mapping from $\Gamma$ onto the set of natural numbers $\mathbb{N}$. The support of a multiset $m=(\Gamma, f)$ is defined as $\operatorname{supp}(m)=\{x \in \Gamma \mid f(x)>0\}$. A multiset is finite (respectively, empty) if its support is a finite (respectively, empty) set. We denote by $\emptyset$ the empty multiset and we denote by $M_{f}(\Gamma)$ the set of all finite multisets over $\Gamma$.

Let $m_{1}=\left(\Gamma, f_{1}\right), m_{2}=\left(\Gamma, f_{2}\right)$ be multisets over $\Gamma$, then the union of $m_{1}$ and $m_{2}$, denoted by $m_{1}+m_{2}$, is the multiset $(\Gamma, g)$, where $g(x)=f_{1}(x)+f_{2}(x)$ for each $x \in \Gamma$. We say that $m_{1}$ is contained in $m_{2}$ and we denote it by $m_{1} \subseteq m_{2}$, if $f_{1}(x) \leq f_{2}(x)$ for each $x \in \Gamma$. The relative complement of $m_{2}$ in $m_{1}$, denoted by $m_{1} \backslash m_{2}$, is the multiset ( $\Gamma$, $g$ ), where $g(x)=f_{1}(x)-f_{2}(x)$ if $f_{1}(x) \geq f_{2}(x)$, and $g(x)=0$ otherwise.

Let us recall that a free tree (tree, for short) is a connected, acyclic, undirected graph. A rooted tree is a tree in which one of the vertices (called the root of the tree) is distinguished from the others. In a rooted tree the concepts of ascendants and descendants are defined in a usual way. Given a node $x$ (different from the root), if the last edge on the (unique) path from the root of the tree to the node $x$ is $\{x, y\}$ (in this case, $x \neq y$ ), then $y$ is the parent of node $x$ and $x$ is a child of node $y$. The root is the only node in the tree with no parent. A node with no children is called a leaf (see [2] for details).

\section{Polarizationless $P$ systems with active membranes}

Let us briefly recall some definitions of P systems models that will be used in the paper (see [9] for details).

A basic transition P system is a membrane system whose rules are of the following forms: evolution, communication, and dissolution. In these systems the size of the membrane structure does not increase, but an exponential workspace (in terms of number of objects) can be constructed in linear time, e.g. via evolution rules of the type $\left[a \rightarrow a^{2}\right]_{h}$. Nevertheless, such capability is not enough to efficiently solve NP-complete problems, unless $\mathbf{P}=\mathbf{N P}$ (see [4] for details).

Replication is one of the most important functions of a cell and, in ideal circumstances, a cell produces two identical copies by division. Bearing in mind that the reactions which take place in a cell are related to membranes, division rules for elementary and non-elementary membranes are considered in the so-called $P$ systems with active membranes. Such variant was first introduced by Gh. Păun [8] and it has associated electrical charges with membranes but the rules are non-cooperative and there are not priorities. Nevertheless, the class of all problems solvable in polynomial time and in a uniform way by means of families of $P$ systems with active membranes which use division for elementary and nonelementary membranes contains class PSPACE and it is contained in class EXP [14]. Thus, in order to provide efficient solutions to computationally hard problems, this framework seems to be too powerful from the computational complexity point of view. 
In this paper, electrical charges are removed from P systems with active membranes. Two different ways of producing an exponential number of membranes in linear time will be considered: division and separation rules (abstractions of mitosis and membrane fission processes, respectively).

\subsection{Polarizationless $P$ system with active membranes: syntax}

Definition 3.1. A polarizationless $\mathrm{P}$ system with active membranes and membrane division of degree $q \geq 2$ is a tuple $\left(\Gamma, H, \mu, \mathcal{M}_{1}, \ldots, \mathcal{M}_{q}, \mathcal{R}, i_{\text {out }}\right)$, where:

- $\Gamma$ is a finite alphabet whose elements are called objects;

- $H$ is a finite alphabet such that $H \cap \Gamma=\emptyset$ whose elements are called labels;

- $\mu$ is a labelled rooted tree consisting of $q$ nodes injectively labelled by elements of $H$ (the leaves of $\mu$ are called elementary membranes and we denote by $i_{\text {skin }}$ the label of the root of $\mu$ );

- $\mathcal{M}_{1}, \ldots, \mathcal{M}_{q}$ are multisets over $\Gamma$;

- $\mathcal{R}$ is a finite set of rules, of the following forms:

$\left(a_{0}\right)[a \rightarrow u]_{h}$, for $h \in H, a \in \Gamma, u \in M_{f}(\Gamma)$ (object evolution).

$\left(b_{0}\right) a[]_{h} \rightarrow[b]_{h}$, for $h \in H \backslash\left\{i_{\text {skin }}\right\}, a, b \in \Gamma$ (send-in communication).

(co) $[a]_{h} \rightarrow b[]_{h}$, for $h \in H, a, b \in \Gamma$ (send-out communication).

$\left(d_{0}\right)[a]_{h} \rightarrow b$, for $h \in H \backslash\left\{i_{\text {out }}, i_{\text {skin }}\right\}, a, b \in \Gamma$ (dissolution).

$\left(e_{0}\right)[a]_{h} \rightarrow[b]_{h}[c]_{h}$, for $h \in H \backslash\left\{i_{\text {out }}, i_{\text {skin }}\right\}, h$ is the label of an elementary membrane, $a, b, c \in \Gamma$ (division for elementary membranes).

$\left(f_{0}\right)\left[[]_{h_{0}}[]_{h_{1}}\right]_{h} \rightarrow\left[[]_{h_{0}}\right]_{h}\left[[]_{h_{1}}\right]_{h}$, where $h \in H \backslash\left\{i_{\text {out }}, i_{\text {skin }}\right\}$ and $h_{0}, h_{1} \in H$ (division for non-elementary membranes).

- $i_{\text {out }} \in H \cup\{e n v\}$, where env $\notin H$, and in the case $i_{\text {out }} \in H$, $i_{\text {out }}$ is the label of a leaf of $\mu$.

Definition 3.2. A polarizationless $\mathrm{P}$ system with active membranes and membrane separation of degree $q \geq 2$ is a tuple $\left(\Gamma, \Gamma_{0}, \Gamma_{1}, H, \mu, \mathcal{M}_{1}, \ldots, \mathcal{M}_{q}, \mathcal{R}, i_{\text {out }}\right)$, where:

- $\left(\Gamma, H, \mu, \mathcal{M}_{1}, \ldots, \mathcal{M}_{q}, i_{\text {out }}\right)$ is as the previous Definition;

- $\left\{\Gamma_{0}, \Gamma_{1}\right\}$ is a partition of $\Gamma$ and $\left\{H_{0}, H_{1}\right\}$ is a partition of $H$;

- $\mathcal{R}$ is a finite set of rules, of the following forms:

$\left(a_{0}\right)[a \rightarrow u]_{h}$, for $h \in H, a \in \Gamma, u \in M_{f}(\Gamma)$ (object evolution).

$\left(b_{0}\right) a[]_{h} \rightarrow[b]_{h}$, for $h \in H, a, b \in \Gamma$ and $h \in H \backslash\left\{i_{\text {skin }}\right\}$ (send-in communication).

(co) $[a]_{h} \rightarrow b[]_{h}$, for $h \in H, a, b \in \Gamma$ (send-out communication).

$\left(d_{0}\right)[a]_{h} \rightarrow b$, for $h \in H \backslash\left\{i_{\text {out }}, i_{\text {skin }}\right\}, a, b \in \Gamma$ (dissolution).

$\left(e_{0}\right)[a]_{h} \rightarrow\left[\Gamma_{0}\right]_{h}\left[\Gamma_{1}\right]_{h}$, for $h \in H \backslash\left\{i_{\text {out }}, i_{\text {skin }}\right\}, h$ is the label of an elementary membrane, $a \in \Gamma$ (separation)

$\left(f_{0}\right)\left[[]_{h_{0}}[]_{h_{1}}\right]_{h} \rightarrow\left[\Gamma_{0}[]_{h_{0}}\right]_{h}\left[\Gamma_{1}[]_{h_{1}}\right]_{h}$, where $h \in H \backslash\left\{i_{\text {out }}, i_{\text {skin }}\right\}, h_{0} \in H_{0}$ and $h_{1} \in H_{1}$ (separation rules for nonelementary membranes).

A polarizationless $\mathrm{P}$ system with active membranes of degree $q \geq 2$, can be viewed as a set of $q$ membranes, labelled by elements of $H$, arranged in a hierarchical structure $\mu$ given by a rooted tree (called membrane structure) whose root is called the skin membrane, such that: (a) $\mathcal{M}_{1}, \ldots, \mathcal{M}_{q}$ represent the finite multisets of objects initially placed in the $q$ membranes of the system; (b) $\mathcal{R}$ is a finite set of rules over $\Gamma$ associated with the labels; and (c) $i_{\text {out }} \in H \cup\{$ en $v\}$ indicates the output region. We use the term region $i$ to refer to membrane $i$ in the case $i \in H$ and to refer to the "environment" of the system in the case $i=e n v$. In these kind of $\mathrm{P}$ systems there are mechanisms, implemented by division rules or separations rules, able to generate an exponential workspace (in terms of number of membranes and objects) in polynomial time. This allows us to describe brute force algorithms in these systems.

\subsection{Polarizationless $P$ system with active membranes: semantics}

An instantaneous description or a configuration $\mathcal{C}_{t}$ at an instant $t$ of a polarizationless $\mathrm{P}$ system with active membranes is described by the following elements: (a) the membrane structure at instant $t$, and (b) all multisets of objects over $\Gamma$ associated with all the membranes present in the system at that moment.

An object evolution rule $[a \rightarrow u]_{h}$ for $h \in H, a \in \Gamma, u \in M_{f}(\Gamma)$ is applicable to a configuration $\mathcal{C}_{t}$ at an instant $t$, if there exists a membrane labelled by $h$ in $\mathcal{C}_{t}$ which contains object $a$. When applying such a rule, object $a$ is consumed and objects from multiset $u$ are produced in that membrane.

A send-in communication rule $a[]_{h} \rightarrow[b]_{h}$ for $h \in H, a, b \in \Gamma$ is applicable to a configuration $\mathcal{C}_{t}$ at an instant $t$, if there exists a membrane labelled by $h$ in $\mathcal{C}_{t}$ such that $h$ is not the label of the root of $\mu$ and its parent membrane contains object $a$. When applying such a rule, object $a$ is consumed from the parent membrane and object $b$ is produced in the corresponding membrane $h$. 
A send-out communication rule $[a]_{h} \rightarrow b[]_{h}$ for $h \in H, a, b \in \Gamma$ is applicable to a configuration $\mathcal{C}_{t}$ at an instant $t$, if there exists a membrane labelled by $h$ in $\mathcal{C}_{t}$ such that it contains object $a$. When applying such a rule, object $a$ is consumed from such membrane $h$ and object $b$ is produced in the parent of such membrane.

A dissolution rule $[a]_{h} \rightarrow b$ for $h \in H \backslash\left\{i_{\text {out }}\right\}, a, b \in \Gamma$ is applicable to a configuration $\mathcal{C}_{t}$ at an instant $t$, if there exists a membrane labelled by $h$ in $\mathcal{C}_{t}$, different from the skin membrane and the output region, such that it contains object $a$. When applying such a rule, object $a$ is consumed, membrane $h$ is dissolved and its objects are sent to the parent (or the first ancestor that has not been dissolved).

A division rule $[a]_{h} \rightarrow[b]_{h}[c]_{h}$ for $h \in H \backslash\left\{i_{\text {out }}\right\}, a, b, c \in \Gamma$, is applicable to a configuration $\mathcal{C}_{t}$ at an instant $t$, if there exists an elementary membrane labelled by $h$ in $\mathcal{C}_{t}$, different from the skin membrane and the output region, such that it contains object $a$. When applying a division rule $[a]_{h} \rightarrow[b]_{h}[c]_{h}$ to a membrane labelled by $h$ in a configuration $\mathcal{C}_{t}$, under the influence of object $a$, the membrane with label $h$ is divided into two membranes with the same label; in the first copy, object $a$ is replaced by object $b$, in the second one, object $a$ is replaced by object $c$; all the other objects are replicated and copies of them are placed in the two new membranes.

A division rule $\left[[]_{h_{0}}[]_{h_{1}}\right]_{h} \rightarrow\left[[]_{h_{0}}\right]_{h}$ [[ $\left.]_{h_{1}}\right]_{h}$ is applicable to a configuration $\mathcal{C}_{t}$ at an instant $t$, if there exists a membrane labelled by $h$ in $\mathcal{C}_{t}$, different from the skin membrane and the output region, which contains a membrane labelled by $h_{0}$ and another membrane labelled by $h_{1}$. When applying such a division rule to a membrane labelled by $h$ in a configuration $\mathcal{C}_{t}$, the membrane with label $h$ is divided into two membranes with the same label; the first copy inherits membrane $h_{0}$ with its contents, and the second copy inherits membrane $h_{1}$ with its contents. Besides, if the membrane labelled by $h$ contains more membranes other than those with the labels $h_{0}, h_{1}$, then such membranes are duplicated so that they become part of the contents of both new copies of the membrane $h$.

A separation rule $[a]_{h} \rightarrow\left[\Gamma_{0}\right]_{h}\left[\Gamma_{1}\right]_{h}$ for $h \in H, a \in \Gamma$, is applicable to a configuration $\mathcal{C}_{t}$ at an instant $t$, if there exists an elementary membrane labelled by $h$ in $\mathcal{C}_{t}$, different from the skin membrane and the output region, such that it contains object $a$. When applying such a rule, the membrane is separated into two membranes with the same label; at the same time, object $a$ is consumed and the multiset of objects contained in membrane $h$ gets distributed: the objects from $\Gamma_{0}$ are placed in the first membrane, those from $\Gamma_{1}$ are placed in the second membrane.

A separation rule $\left[[]_{h_{0}}[]_{h_{1}}\right]_{h} \rightarrow\left[\Gamma_{0}[]_{h_{0}}\right]_{h}\left[\Gamma_{1}[]_{h_{1}}\right]_{h}$, where $h, h_{0}, h_{1}$ are labels such that $h_{0} \in H_{0}$ and $h_{1} \in H_{1}$, is applicable to a configuration $\mathcal{C}_{t}$ at an instant $t$, if there exists a membrane labelled by $h$ in $\mathcal{C}_{t}$, different from the skin membrane and the output region, such that it contains a membrane labelled by $h_{0}$ and another membrane labelled by $h_{1}$. When applying such a separation rule to a membrane labelled by $h$ in a configuration $\mathcal{C}_{t}$, that membrane is separated into two membranes with the same label, in such a way that the contents (multiset of objects and inner membranes) are distributed as follows: The first membrane receives the multiset of objects from $\Gamma_{0}$, and all inner membranes whose label belongs to $H_{0}$; and the second membrane receives the multiset of objects from $\Gamma_{1}$, and all inner membranes whose label belongs to $H_{1}$.

In polarizationless $\mathrm{P}$ systems with active membranes, the rules are applied according to the following principles:

- The rules associated with membranes labelled with $h$ are used for all copies of this membrane.

- At one transition step, one object can be used by only one rule (chosen in a non-deterministic way).

- At one transition step, a membrane can be the subject of only one rule of types $\left(b_{0}\right)-\left(f_{0}\right)$, and then it is applied at most once.

- Object evolution rules can be simultaneously applied to a membrane with one rule of types $\left(b_{0}\right)-\left(f_{0}\right)$. Object evolution rules are applied in a maximally parallel manner.

- If at the same time a membrane labelled with $h$ is divided by a rule of type $\left(e_{0}\right)$ or $\left(f_{0}\right)$ and there are objects in this membrane which evolve by means of rules of type $\left(a_{0}\right)$, then we suppose that first the evolution rules of type $\left(a_{0}\right)$ are used, changing the objects, and then the division (or the separation) is produced. Of course, this process takes only one transition step.

- The skin membrane and the output membrane can never get divided, separated, nor dissolved.

Let us notice that in these kind of $\mathrm{P}$ systems the environment plays a passive role in the following sense: along any computation, the environment only can receive objects from the system but it cannot send objects into the system.

\section{Polynomial complexity classes of recognizer membrane systems}

In what follows, a membrane system denotes a $\mathrm{P}$ system of any of the different variants considered in the previous section. The concept of recognizer membrane system is defined as usual (see [11] for details). It is worth noting that in these systems the working alphabet $\Gamma$ has two distinguished objects yes and no, there exists an input alphabet $\sigma$, the initial multisets of the system are multisets are multisets over $\Gamma \backslash \Sigma$, the output region is the environment, all computations halt, and for every computation, then either object yes or object no (but not both) must have been released into the environment, and only at the last step of the computation.

If $\Pi$ is a recognizer membrane system then for each multiset $m$ over the input alphabet $\Sigma$, we denote by $\Pi+m$ the system $\Pi$ where at the initial configuration, the multiset $m$ has been added to the input membrane. Thus, we have an initial 
configuration associated with each input multiset $m$ over $\Sigma$ in this kind of systems. Any computation of $\Pi+m$ starts from such an initial configuration.

We denote by $\mathcal{D} \mathcal{A} \mathcal{M}^{0}(\gamma, \delta)$ where $\gamma \in\{-d,+d\}$ and $\delta \in\{-n,+n\}$, the class of all recognizer polarizationless $\mathrm{P}$ systems with active membranes and division rules. The meaning of parameters $\gamma$ and $\delta$ is the following:

- if $\gamma=+d$ (resp. $\gamma=-d$ ) then dissolution rules are permitted (resp. forbidden);

- if $\delta=+n$ (resp. $\delta=-n$ ) then division rules for elementary and non-elementary membranes are permitted (resp. only division rules for elementary membranes are permitted).

In a similar way, notation $\mathcal{S A M}^{0}(\gamma, \delta)$ is considered when we use separation rules instead of division rules.

Next, let us recall the concept of efficient solvability by means of a family of recognizer membrane systems (see [10] for more details).

Definition 4.1. Let $\mathcal{R}$ be a class of recognizer membrane systems. We say that a decision problem $X$ is solvable in polynomial time by a family $\Pi=\{\Pi(n) \mid n \in \mathbb{N}\}$ of systems from $\mathcal{R}$, in a uniform way, denoted by $X \in$ PMC $_{\mathcal{R}}$, if the following hold:

- the family $\Pi$ is polynomially uniform by Turing machines;

- there exists a pair (cod,s) of polynomial-time computable functions over $I_{X}$ such that:

- for each instance $u \in I_{X}, s(u)$ is a natural number and $\operatorname{cod}(u)$ is an input multiset of the system $\Pi(s(u))$;

- for each $n \in \mathbb{N}, s^{-1}(n)$ is a finite set;

- the family $\Pi$ is polynomially bounded, sound and complete with regard to $(X, \operatorname{cod}, s)$.

The polynomial complexity class $\mathbf{P} \mathbf{M} \mathbf{C}_{\mathcal{R}}$ is closed under polynomial-time reduction and under complement [12].

\section{Minimal cooperation in object evolution rules of polarizationless $P$ systems with active membranes}

Let us recall that polarizationless $\mathrm{P}$ systems with active membranes are non-cooperative systems, that is, the left-hand side of the rules of these systems has only one object (the objects do not directly interact). Moreover, with the exception of object evolution rules, single objects are always transformed into single objects (the two objects produced by a division rule are placed in two different compartments/membranes).

Let us also recall that by using the dependency graph technique it has been shown that if dissolution rules are forbidden, then only tractable problems can be solved in an efficient way by families of polarizationless $\mathrm{P}$ systems with active membranes even using division rules for non-elementary membranes (see [3] for details), that is, $\mathbf{P M C}_{\mathcal{D} \mathcal{A M}}{ }^{0}(-d,+n)=\mathbf{P}_{\text {. }}$ It is worth pointing out that in polarizationless $\mathrm{P}$ systems with active membranes and without dissolution rules, the term "non-elementary membrane" is static, that is, in any configuration of any computation of the system, a membrane is elementary if and only if it is elementary at the initial configuration of the system (a "non-elementary membrane" never can "evolve" to an "elementary membrane").

Recent works have explored which syntactical ingredients could be added to the framework $\mathcal{D} \mathcal{A} \mathcal{M}^{0}(-d,-n)$ in order to reach computational efficiency. Specifically, it has been shown that incorporating some kind of minimal cooperation (called bounded minimal cooperation) in object evolution rules, computationally hard problems can be solved by families of polarizationless $\mathrm{P}$ systems with active membranes without dissolution and making use only of division for elementary membranes [15]. This framework is denoted by $\mathcal{D} \mathcal{A} \mathcal{M}_{b m c}^{0}(-d,-n)$.

When separation rules are considered instead of division rules as a mechanism to provide an exponential workspace in polynomial time, the dependency graph technique can be used to prove that if dissolution rules are forbidden, then only tractable problems can be solved in an efficient way by families of polarizationless $\mathrm{P}$ systems with active membranes and with separation rules (see [16] for details), that is, $\mathbf{P M C}_{\mathcal{S} \mathcal{A} \mathcal{M}^{0}(-d,+n)}=\mathbf{P}$. In this paper, the efficiency of systems from $\mathcal{S} \mathcal{A} \mathcal{M}^{0}(-d,+n)$ when bounded minimal cooperation in object evolution rules are considered instead of non-cooperative rules, is analyzed. Specifically, we will show that the role of dissolution rules is irrelevant when bounded minimal cooperation in object evolution rules are used, that is, $\mathbf{P M C}_{\mathcal{S A M}_{b m c}^{0}(+d,+n)}=\mathbf{P}$.

Definition 5.1. A polarizationless $P$ system with active membranes, with division or separation rules, is said to have bounded minimal cooperation in object evolution rules, if the object evolution rules are of the type [u $\rightarrow v]_{h}$, for $1 \leq|v| \leq|u| \leq 2$.

Let us notice that in these systems the left-hand side of object evolution rules is, at most, two. Thus, the cooperation in object evolution rules is minimal. Besides, the number of objects in the right-hand side is lesser or equal to the number of objects in the left-hand side. Therefore, by applying these types of rules the total number of objects of the system does not increase.

We denote by $\mathcal{D} \mathcal{A} \mathcal{M}_{b m c}^{0}(\gamma, \delta)$ (resp. $\mathcal{S} \mathcal{A} \mathcal{M}_{b m c}^{0}(\gamma, \delta)$ ) the class of all recognizer polarizationless $\mathrm{P}$ systems with active membranes, bounded minimal cooperation in object evolution rules and division (resp. separation) rules. 
Let us notice that standard notation in the literature referring to polarizationless $\mathrm{P}$ systems with active membranes $\left(\mathcal{A M}^{0}(\gamma, \delta)\right)$ corresponds, within this new notation, to the class $\mathcal{D} \mathcal{A} \mathcal{M}^{0}(\gamma, \delta)$.

\section{Limits on efficient computations in $\mathcal{S} \mathcal{A} \mathcal{M}_{b m c}^{0}(+d,+n)$}

In this section we study the computational efficiency of polarizationless $\mathrm{P}$ systems with active membranes, dissolution rules and minimal cooperation when separation rules (for elementary and non-elementary membranes) are considered as a mechanism to generate an exponential workspace in linear time. Specifically, we will show that these kind of P systems can only solve problems in class $\mathbf{P}$ in an efficient way. The proof is inspired on a similar result, obtained in the framework of cell-like P systems with symport/antiport rules and cell separation [5].

Let $\Pi=\left(\Gamma, \Gamma_{0}, \Gamma_{1}, \Sigma, H, H_{0}, H_{1}, \mu, \mathcal{M}_{1}, \ldots, \mathcal{M}_{q}, \mathcal{R}, i_{\text {in }}, i_{\text {out }}\right)$ be a recognizer P system from $\mathcal{S} \mathcal{A} \mathcal{M}_{b m c}^{0}(+d,+n)$. In what follows we use the concepts of notation from [13].

- We denote by $p(i)$ (resp., $\operatorname{ch}(i)$ ) the label of the parent (resp., a child) of the membrane labelled by $i$, the parent of the skin membrane is the environment (we write $p(1)=0$ ). We denote by $\mathcal{R}_{E}$ (resp., $\mathcal{R}_{C}, \mathcal{R}_{D}$ and $\mathcal{R}_{S}$ ) the set of evolution rules (resp., communication, dissolution and separation rules) of $\Pi$. We will fix total orders in $\mathcal{R}_{E}, \mathcal{R}_{C}, \mathcal{R}_{D}$ and $\mathcal{R}_{S}$.

- Let $\mathcal{C}$ be a computation of $\Pi$, and $\mathcal{C}_{t}$ an arbitrary configuration of $\mathcal{C}$. With respect to the number of objects of the system, let us notice that by applying a single rule, this number remains unchanged or decreases by one. Thus, the total number of objects in $\mathcal{C}_{t}$ is, at most, $M$, being $M=\left|\mathcal{M}_{0}+\ldots+\mathcal{M}_{q}\right|$.

With respect to the number of membranes of the system, by applying a separation rule for elementary membranes, an object is removed from the system, no new objects are produced and a new membrane is created. Thus, at most $M$ membranes can be produced by means of this process. Also, by applying a separation rule for non-elementary membranes, the number of objects remains unchanged but a new membrane is created (when such a rule is applied to a non-elementary membrane, it cannot be applied to that membrane anymore). In this way, no more than $q-2$ new membranes can be generated. Consequently, $q+M+(q-2)=M+2 q-2$ is an upper bound of the total number of membranes at $\mathcal{C}_{t}$.

- In order to identify the membranes created by the application of a separation rule, we modify the labels of the new membranes in the following recursive manner:

- The label of a membrane will be a pair $(i, \sigma)$ where $0 \leq i \leq q$ and $\sigma \in\{0,1\}^{*}$. At the initial configuration, the labels of the membranes are $(1, \lambda), \ldots,(q, \lambda)$. The label of the environment is denoted by $(0, \lambda)$.

- If a separation rule is applied to a membrane labelled by $(i, \sigma)$, then the new created membranes will be labelled by $(i, \sigma 0)$ and $(i, \sigma 1)$, respectively. Membrane $(i, \sigma 0)$ will only contain the objects of membrane $(i, \sigma)$ which belong to $\Gamma_{0}$, and membrane $(i, \sigma 1)$ will only contain the objects of membrane $(i, \sigma)$ which belong to $\Gamma_{1}$. Only elementary membranes can be separated, so if a membrane $i$ is non-elementary then we denote it by the label $(i, \lambda)$.

- If an object evolution rule or a communication rule is applied to a membrane labelled by $(i, \sigma)$, then after the application of the rule, the membrane keeps its label.

- Let us notice that the number of labels we need to identify all membranes appearing along any computation of a $\mathrm{P}$ system from $\mathcal{S} \mathcal{A M}_{b m c}^{0}(+d,+n)$ is of the order $O(M+q)$.

- A configuration $\mathcal{C}_{t}$ of a P system from $\mathcal{S A M}_{b m c}^{0}(+d,+n)$ is described by the current membrane structure and the multisets of labelled objects of the type

$$
\left\{(a, i, \sigma): a \in \Gamma, 0 \leq i \leq q, \sigma \in\{0,1\}^{*}\right\}
$$

The expression $(a, i, \sigma) \in \mathcal{C}_{t}$ means that object $a$ belongs to membrane labelled by $(i, \sigma)$.

- Let $r=[a b \rightarrow c]_{h} \in \mathcal{R}$ be an object evolution rule of $\Pi$. We denote by $n \cdot L H S(r,(i, \sigma)), n \in \mathbf{N}$, the multiset of labelled objects $(a, i, \sigma)^{n}(b, i, \sigma)^{n}$ We denote by $n \cdot R H S(r,(i, \sigma))$ the multiset of labelled objects $(c, i, \sigma)^{n}$ produced by applying $n$ times rule $r$ over membrane $(i, \sigma)$. Similarly these concepts are defined for object evolution rules of the forms $[a b \rightarrow c d]_{h}$ and $[a \rightarrow c]_{h}$.

- Let $r=[a]_{h} \rightarrow b[]_{h} \in \mathcal{R}$ be a send-out communication rule of $\Pi$. We denote by $L H S(r,(i, \sigma))$ the labelled object $(a, i, \sigma)$. We denote by $\operatorname{RHS}(r,(i, \sigma))$ the labelled object $(b, p(i), \tau)$ produced by applying rule $r$ over membrane $(i, \sigma)$, where $(p(i), \tau)$ is the parent of membrane $(i, \sigma)$.

- Let $r=a[\quad]_{h} \rightarrow[b]_{h} \in \mathcal{R}$ be a send-in communication rule of $\Pi$. We denote by $L H S(r,(i, \sigma))$ the labelled object $(a, p(i), \tau)$, where $(p(i), \tau)$ is the parent of membrane $(i, \sigma)$. We denote by $R H S(r,(i, \sigma))$ the labelled object $(b, i, \sigma)$ produced by applying rule $r$ over membrane $(i, \sigma)$.

- Let $\mathcal{C}_{t}$ is a configuration of $\Pi$, we denote by $\mathcal{C}_{t}+\left\{(x, i, \sigma) / \sigma^{\prime}\right\}$ the multiset obtained by replacing in $\mathcal{C}_{t}$ every occurrence of $(x, i, \sigma)$ by $\left(x, i, \sigma^{\prime}\right)$. Besides, $\mathcal{C}_{t}+m$ (resp., $\mathcal{C}_{t} \backslash m$ ) is used to denote that a multiset $m$ of labelled objects is added (resp., removed) to the configuration.

Next, we provide a deterministic algorithm $\mathcal{A}$ working in polynomial time that receives as input a recognizer $\mathrm{P}$ system $\Pi$ from $\mathcal{S} \mathcal{A} \mathcal{M}_{b m c}^{0}(+d,+n)$ together with an input multiset $m$ of $\Pi$. Then algorithm $\mathcal{A}$ reproduces the behaviour of a single computation of such system. 
The pseudocode of the algorithm $\mathcal{A}$ is described as follows:

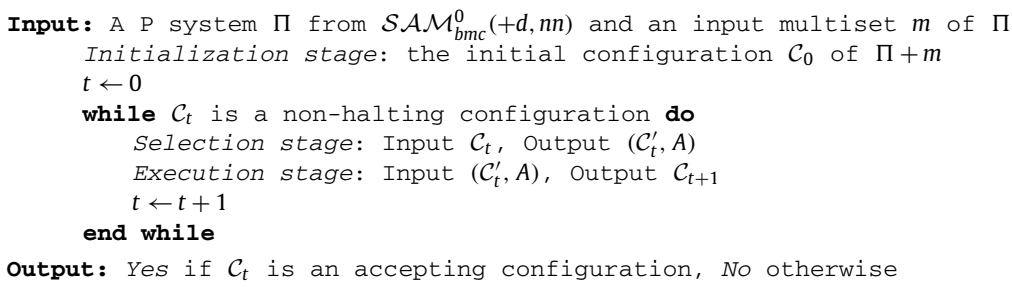

The selection stage and the execution stage implement a transition step of a recognizer P system $\Pi$. Specifically, the selection stage receives as input a configuration $\mathcal{C}_{t}$ of $\Pi$ at an instant $t$. The output of this stage is a pair $\left(\mathcal{C}_{t}^{\prime}, A\right)$, where $A$ encodes a multiset of rules selected to be applied to $\mathcal{C}_{t}$, and $\mathcal{C}_{t}^{\prime}$ is the configuration obtained from $\mathcal{C}_{t}$ once the labelled objects corresponding to the application of rules from $A$ have been consumed. The execution stage receives as input the output $\left(\mathcal{C}_{t}^{\prime}, A\right)$ of the selection stage, and the output is the next configuration $\mathcal{C}_{t+1}$ of $\mathcal{C}_{t}$. Specifically, at this stage, configuration $\mathcal{C}_{t}^{\prime}$ yields configuration $\mathcal{C}_{t+1}$ by adding the labelled objects produced by the application of rules from $A$.

Next, selection stage and execution stage are described in detail.

\section{Selection stage.}

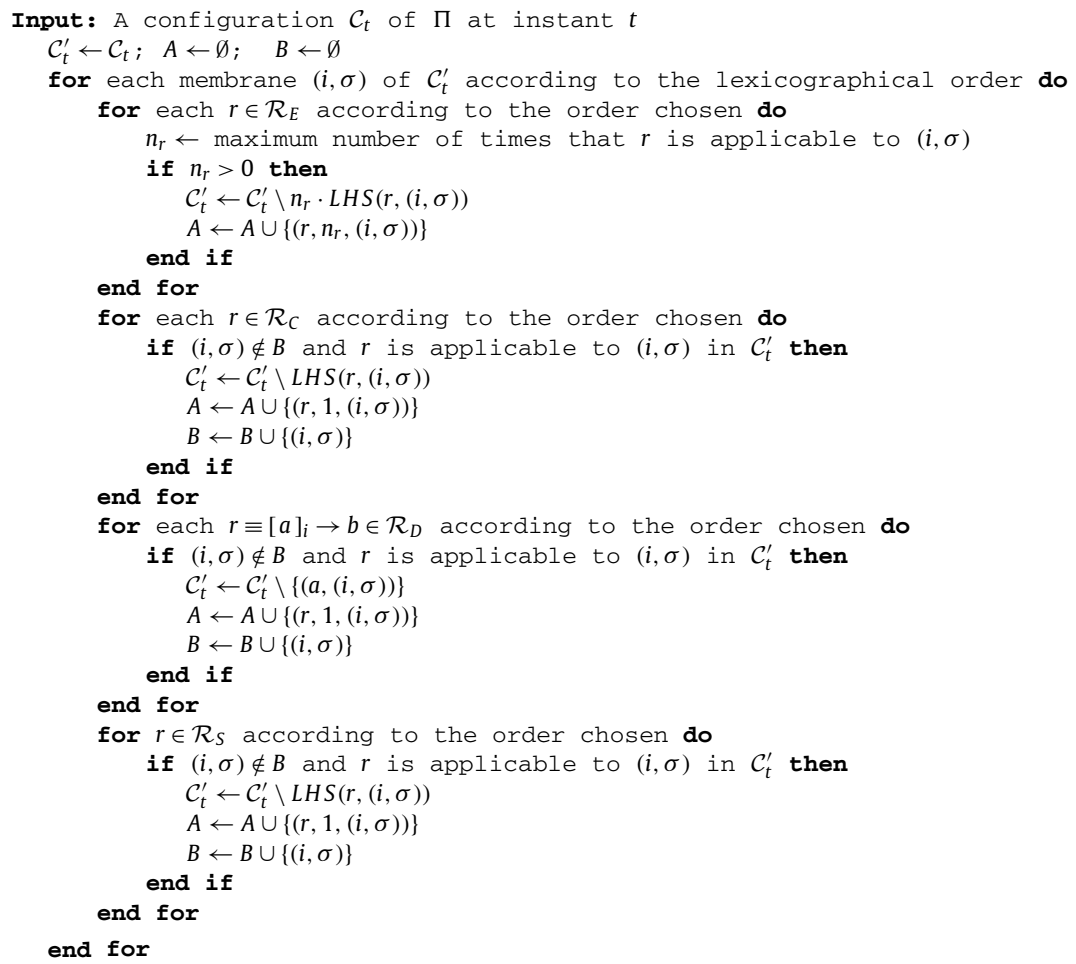

This algorithm is deterministic and works in polynomial time. Indeed, the cost in time is polynomial in the size of $\Pi$ because the number of cycles of the external main for loop is of order $O(M+q)$, and the number of cycles of the three internal main for loops are of order $O(|R|)$. Besides, the cost of each internal loops is of the order $O(M+q)$.

Let us notice that the number of tuples in set $A$ is of the order $O(M)$ because each object in the system can be involved in, at most, one rule and at any configuration $\mathcal{C}_{t}$ the total number of objects is upper bounded by $M$. In set $A$ an order is considered in a natural way (a product order concerning the rules, natural numbers and labels).

In order to complete the simulation of a computation step of the system $\Pi$, the execution stage takes care of the effects of applying the rules selected in the previous stage: updating the objects according to the RHS of the rules.

\section{Execution stage.}

Input: The output $\mathcal{C}_{t}^{\prime}$ and $A$ of the selection stage

for each $\left(r, n_{r},(i, \sigma)\right) \in A$ according to the order chosen do

if $r \in \mathcal{R}_{E}$ then

$\mathcal{C}_{t}^{\prime} \leftarrow \mathcal{C}_{t}^{\prime}+n_{r} \cdot R H S(r,(i, \sigma))$ 


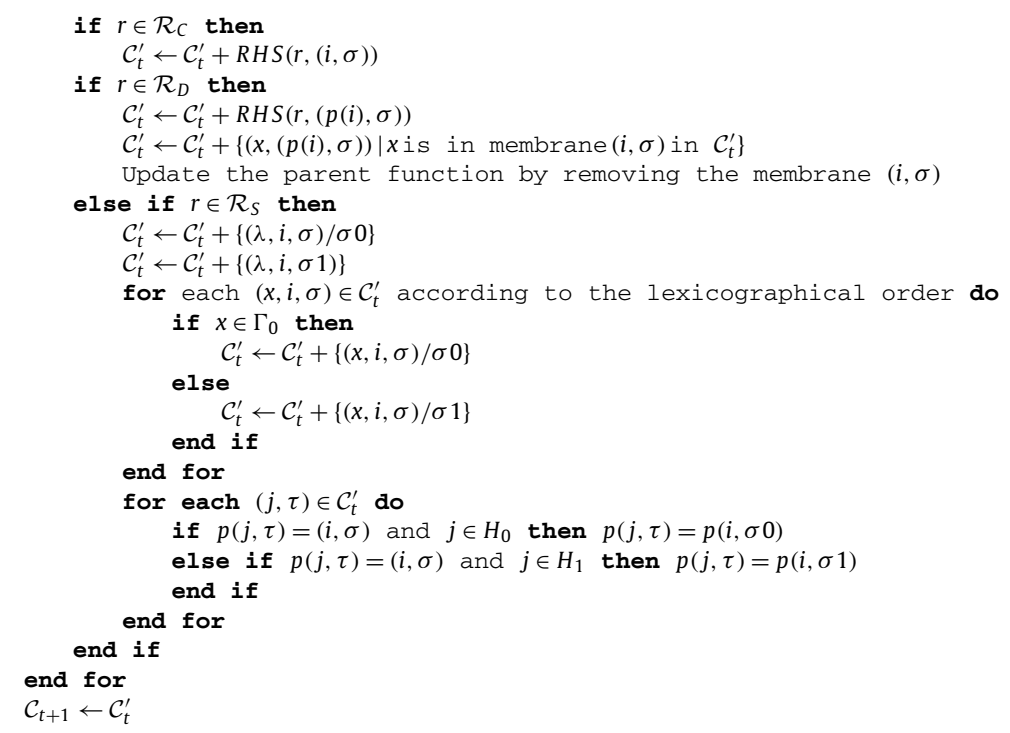

This algorithm is deterministic and works in polynomial time. Indeed, on the one hand, the number of cycles of the main for loop is of order $O(M)$. On the other hand, each cycle of the main for loop takes $O(|R|)$ steps plus the number of steps spend by the two secondary for loops: the first takes $O(M(M+q))$ steps and the second takes $O(M+q)$ steps.

Theorem 1. $\mathbf{P}=\mathbf{P M C}_{\mathcal{S A M}} \mathcal{M}_{b m c}^{0}(+d,+n)$.

Proof. It suffices to prove that $\mathbf{P M C}_{\mathcal{S A M}_{b m c}^{0}(+d,+n)} \subseteq \mathbf{P}$. For that, let $X=\left(I_{X}, \theta_{X}\right)$ be a decision problem in PMC $_{\mathcal{S} \mathcal{A M}_{b m c}^{0}(+d,+n)}$. Let $\{\Pi(n) \mid n \in \mathbb{N}\}$ be a family of P systems from $\mathcal{S A M}_{b m c}^{0}(+d,+n)$ solving $X$, according to Definition 4.1. Let $(\operatorname{cod}, s)$ be a polynomial encoding associated with that solution. Let us recall that instance $u \in I_{X}$ of the problem $X$ is processed by the system $\Pi(s(u))+\operatorname{cod}(u)$.

Let us consider the following deterministic algorithm $\mathcal{A}^{\prime}$ :

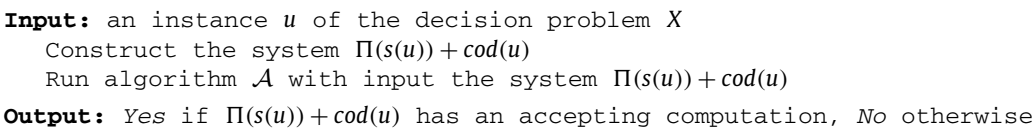

Given an instance $u$ of the decision problem $X=\left(I_{X}, \theta_{X}\right)$, the following assertions are equivalent:

1. $\theta_{X}(u)=1$, that is, the answer of problem $X$ to instance $u$ is affirmative.

2. Every computation of $\Pi(s(u))+\operatorname{cod}(u)$ is an accepting computation.

3. The output of the algorithm with input $u$ is Yes.

Therefore, algorithm $\mathcal{A}^{\prime}$ provides a solution of the decision problem $X$. Bearing in mind that $\mathcal{A}^{\prime}$ works in polynomial time, we finally deduce that $X \in \mathbf{P}$.

\section{From distribution to replication in membrane systems with bounded minimal cooperation}

In this Section, a new frontier of the efficiency is obtained in the framework of polarizationless P systems with active membranes which makes use of bounded minimal cooperation in object evolution rules.

On the one hand, in the previous Section we have shown that only problems in class $\mathbf{P}$ can be solved in polynomial time by families of polarizationless $\mathrm{P}$ systems with active membranes and with separation rules which makes use of bounded minimal cooperation in object evolution rules, that is, $\mathbf{P}=\mathbf{P M C}_{\mathcal{S} \mathcal{A} \mathcal{M}_{b m c}^{0}(+d,+n)}$.

On the other hand, in [15] a uniform polynomial time solution to the SAT problem by a family of polarizationless P systems with active membranes and with division rules which makes use of bounded minimal cooperation in object evolution rules, has been provided, that is, $\mathbf{N P} \cup \mathbf{c o}-\mathbf{N P} \subseteq \mathbf{P M C}_{\mathcal{D} \mathcal{A} \mathcal{M}_{b m c}^{0}(-d,-n)}$.

Consequently, assuming that $\mathbf{P} \neq \mathbf{N P}$, a new tractability borderline between efficiency and non-efficiency has been obtained in the framework of polarizationless P systems with active membranes and without dissolution which makes use of bounded minimal cooperation in object evolution rules, in terms of the mechanism used to generate an exponential 
workspace in polynomial time: passing from separation rules to division rules amounts to passing from non-efficiency to efficiency.

\section{Conclusions}

The classical definition of polarizationless $\mathrm{P}$ systems with active membranes makes use of non-cooperative rules and their object evolution rules are of the form $[a \rightarrow u]_{h}$, where $a$ is an object of the working alphabet and $u$ is a finite multiset of objects. In that context, the capability of these membrane systems to create an exponential workspace in polynomial time is implemented by means of division rules (for both elementary and non-elementary membranes). It is well known [3] that only tractable problems can be solved in an efficient way by families of such kind of P systems which do not make use of dissolution rules even if division rules for elementary and non-elementary membranes is allowed, that is, $\mathbf{P}=\mathbf{P M C}_{\mathcal{D} \mathcal{A M}}{ }_{(-d,+n)}$. Besides, in this context dissolution rules play a (surprisingly) very important role, because when this kind of rules are permitted then PSPACE-complete problems can be solved efficiently [1], that is PSPACE $\subseteq$ PMC $_{\mathcal{D} \mathcal{A} \mathcal{M}^{0}(+d,+n)}$.

In this paper, two variants are considered. First, by using separation rules inspired on the membrane fission mechanism, instead of division rules in order to create an exponential workspace in polynomial time. Second, bounded minimal cooperation in object evolution rules is considered in polarizationless P systems with active membranes making use of division or separation rules. Object evolution rules with bounded minimal cooperation are of the type $[u \rightarrow v]_{h}$, where $u, v$ are finite multisets of objects such that $1 \leq|v| \leq|u| \leq 2$. The corresponding family of recognizer systems are denoted by $\mathcal{D} \mathcal{A} \mathcal{M}_{b m c}^{0}(\gamma, \delta)$ and $\mathcal{S} \mathcal{A} \mathcal{M}_{b m c}^{0}(\gamma, \delta)$, respectively.

On the one hand, the computational efficiency of systems from $\mathcal{D} \mathcal{A} \mathcal{M}_{b m c}^{0}(-d,-n)$ was shown in [15] by giving a polynomial time solution to the SAT problem by means of a family of such systems. On the other hand, in this paper the limits on efficient computation in $\mathcal{S} \mathcal{A M}_{b m c}^{0}(+d,+n)$ has been established, in the sense that only problems in class $\mathbf{P}$ can be solved efficiently by families of such kind of membrane systems. That is, whereas using division rules but not dissolution rules, bounded minimal cooperation allows us to reach the efficiency, using separation rules (even adding dissolution rules too) that requirement is not enough to achieve efficiency.

Therefore, assuming that $\mathbf{P} \neq \mathbf{N P}$, in the framework of polarizationless $\mathrm{P}$ systems with active membranes, without dissolution and bounded minimal cooperation in object evolution rules, a new frontier of the efficiency has been obtained when division rules are used instead of separation rules.

These results confirm two interesting aspects related with complexity point of view:

- The strength of the replication of objects (division) with respect to the distribution of objects (separation).

- The irrelevant role played by dissolution rules when bounded minimal cooperation is allowed.

As future work, we propose several research lines related to the computational efficiency of new variants of polarizationless $\mathrm{P}$ systems with active membranes.

(a) What about the complexity classes of polarizationless P systems with active membranes and separation which make use of classical object evolution rules?

(b) What about the complexity classes of polarizationless $\mathrm{P}$ systems with active membranes and separation which incorporate minimal cooperation in object evolution rules, removing the restriction about the length of the right-hand side of the rules?

(c) Membrane systems that incorporate an environment with an active role in polarizationless $P$ systems with active membranes through a distinguished alphabet $\mathcal{E}$ similarly to the considered in cell-like $\mathrm{P}$ systems with symport/antiport rules (see $[5,6]$ for details). Then two kind of semantics can be considered: the classical semantics of active membranes or a semantics based on maximal parallelism of the rules except for division or separation rules. Is the role of the environment relevant from a computational complexity point of view?

\section{References}

[1] A. Alhazov, M.J. Pérez-Jiménez, Uniform solution of QSAT using polarizationless active membranes, Lecture Notes in Comput. Sci. 4664 (2007) 122-133.

[2] T.H. Cormen, C.E. Leiserson, R.L. Rivest, An Introduction to Algorithms, The MIT Press, Cambridge, Massachusetts, 1994.

[3] M.A. Gutiérrez-Naranjo, M.J. Pérez-Jiménez, A. Riscos-Núñez, F.J. Romero-Campero, On the power of dissolution in P systems with active membranes, Lecture Notes in Comput. Sci. 3850 (2006) 224-240.

[4] M.A. Gutiérrez-Naranjo, M.J. Pérez-Jiménez, A. Riscos-Núñez, F.J. Romero-Campero, A. Romero-Jiménez, Characterizing tractability by cell-like membrane systems, in: K.G. Subramanian, K. Rangarajan, M. Mukund (Eds.), Formal Models, Languages and Applications, World Scientific, Singapore, 2006, pp. $137-154$.

[5] L.F. Macías-Ramos, M.J. Pérez-Jiménez, A. Riscos-Núñez, L. Valencia-Cabrera, Membrane fission versus cell division: when membrane proliferation is not enough, Theoret. Comput. Sci. 608 (2015) 57-65.

[6] L.F. Macías-Ramos, B. Song, L. Valencia-Cabrera, L. Pan, M.J. Pérez-Jiménez, Membrane fission: a computational complexity perspective, Complexity (2015), https://doi.org/10.1002/cplx.21691, online version.

[7] Gh. Păun, Attacking NP-complete problems, in: I. Antoniou, C. Calude, M.J. Dinneen (Eds.), Unconventional Models of Computation, UMC'2K, SpringerVerlag, 2000, pp. 94-115. 
[8] Gh. Păun, P systems with active membranes: attacking NP-complete problems, J. Autom. Lang. Comb. 6 (1) (2001) 75-90.

[9] Gh. Păun, G. Rozenberg, A. Salomaa (Eds.), The Oxford Handbook of Membrane Computing, Oxford University Press, Oxford, 2010.

[10] M.J. Pérez-Jiménez, An approach to computational complexity in Membrane Computing, Lecture Notes in Comput. Sci. 3365 (2005) 85-109.

[11] M.J. Pérez-Jiménez, A. Romero-Jiménez, F. Sancho-Caparrini, Complexity classes in cellular computing with membranes, Nat. Comput. 2 (3) (2003) $265-285$.

[12] M.J. Pérez-Jiménez, A. Romero-Jiménez, F. Sancho-Caparrini, A polynomial complexity class in P systems using membrane division, J. Autom. Lang. Comb. 11 (4) (2006) 423-434, A preliminary version in: E. Csuhaj-Varjú, C. Kintala, D. Wotschke, Gy. Vaszil (Eds.), Proceedings of the Fifth International Workshop on Descriptional Complexity of Formal Systems, DCFS 2003, July 12-14, 2003, Budapest, Hungary, 2003, pp. 284-294.

[13] M.J. Pérez-Jiménez, A computational complexity theory in membrane computing (invited talk), in: Membrane Computing, 10th International Workshop, WMC 2009, Curtea de Arges, Romania, August 24-27, 2009, in: Lecture Notes in Comput. Sci., vol. 5957, 2010, pp. 125-148, Revised Selected and Invited Papers.

[14] A.E. Porreca, G. Mauri, C. Zandron, Complexity classes for membrane systems, Inform. Théor. Appl. 40 (2) (2006) 141-162.

[15] L. Valencia-Cabrera, D. Orellana-Martín, M.A. Martínez-del-Amor, A. Riscos-Núñez, M.J. Pérez-Jiménez, Polarizationless P systems with active membranes: computational complexity aspects, J. Autom. Lang. Comb. 21 (1-2) (2016) 101-117.

[16] L. Valencia-Cabrera, D. Orellana-Martín, M.A. Martínez-del-Amor, A. Riscos-Núñez, M.J. Pérez-Jiménez, Computational efficiency of minimal cooperation and distribution in polarizationless P systems with active membranes, Fund. Inform. (2016), in press. 